\title{
Vibro-Acoustic Tissue Mammography
}

\author{
Mostafa Fatemi*, Member, IEEE, Lester E. Wold, Azra Alizad, and James F. Greenleaf, Fellow, IEEE
}

\begin{abstract}
A novel method for detection and imaging of microcalcifications in breast tissue is presented. The method, called vibro-acoustography, uses the radiation force of ultrasound to vibrate tissue at low $(\mathrm{kHz})$ frequency and utilizes the resulting response to produce images that are related to the hardness of the tissue. The method is tested on human breast tissues. The resulting vibro-acoustographic images are in agreement with corresponding $X$-ray mammography images of the specimens. The existence of microcalcifications in locations indicated by vibro-acoustography is confirmed by histology. Microcalcifications as small as $110 \mu \mathrm{m}$ in diameter are detected by this method. Resulting vibro-acoustographic images show microcalcifications with high contrast with respect to the background soft tissue. Structures such as dense sclerotic tissue do not seem to interfere with detection of microcalcifications.
\end{abstract}

Index Terms-Breast, mammography, microcalcifications, ultrasound, vibro-acoustography.

\section{INTRODUCTION}

$\mathbf{X}$ -RAY mammography is currently the only imaging modality clinically used for detection of breast microcalcifications. The widespread use of screening mammography has resulted in the increased detection of microcalcifications [1]. A wide spectrum of breast lesions is associated with microcalcifications, ranging from benign (fibrocystic changes, vascular changes, fat necrosis) to malignant [2]. The mammographic detection of microcalcifications frequently results in additional diagnostic studies, including: spot magnification mammographic views, stereotaxic biopsy and wire-localized biopsies. Follow-up of microcalcifications that are not biopsied, or that are benign by stereotaxic biopsy, may require additional mammograms, e.g., at six-month intervals. This results in significant additional costs, discomfort, anxiety, and some increase in X-ray exposure [1]. Recent changes in American Cancer Society (ACS) and National Cancer Institute (NCI) screening guidelines for women in their forties will increase both the number of mammograms and the number of detected microcalcifications, with resultant increased costs and morbidity. The ACS recommends annual mammography for

Manuscript received April 30, 2001; revised November 20, 2001. This work was supported by the Army Medical Research and Material Command under Grant DAMD 17-98-1-8121. The Associate Editor responsible for coordinating the review of this paper and recommending its publication was M. Insana. Asterisk indicates corresponding author.

*M. Fatemi is with the Basic Ultrasound Research Laboratory, Department of Physiology and Biophysics, Mayo Clinic and Mayo Foundation, Rochester, MN 55905 USA (e-mail: fatemi.mostafa@mayo.edu).

L. E. Wold is with the Laboratory of Medicine and Biology, Mayo Clinic and Mayo Foundation, Rochester, MN 55905 USA

A. Alizad and J. F. Greenleaf are with the Basic Ultrasound Research Laboratory, Department of Physiology and Biophysics, Mayo Clinic and Mayo Foundation, Rochester, MN 55905 USA.

Publisher Item Identifier S 0278-0062(02)01043-1. women beginning at age 40. In women at "high" risk for breast cancer surveillance may begin at age 35 [3].

There exist subsets of women for whom mammographic screening and follow-up is limited by reduced mammographic sensitivity. Fibrocystic change is the most common benign condition of the breast, with the greatest incidence in women 30-50 years old [4]. The ACS estimates that about half of all women in America have fibrocystic breasts and many of them have very radiologically dense breast tissue. For this group, the failure rate to detect cancer on their mammogram may be as high as $15 \%-25 \%$ [5], [6]. Mammography is more effective in detecting occult malignancy as age increases and the breast becomes more fatty. It is the density of the breast on film screen mammography that most directly affects the risk for false-negative and false-positive interpretation [7], [8]. Several factors affect breast density. Age is the most significant factor, with more premenopausal women, especially in the 40- to 49-year age group, having breasts that are denser. For premenopausal women mammographic sensitivity may be increased by scheduling evaluation the first two weeks of their cycle corresponding with the follicular phase [9], [10]. Pregnant or lactating women may have extremely dense breast tissue, which may affect the sensitivity of mammography; also these conditions are a relative contraindication for X-ray mammography and, thus, limit its usefulness. Some studies have shown hormone replacement therapy, commonly used in postmenopausal patients, reduces the sensitivity of mammographic screening [11]-[13]. The sensitivity or accuracy of film screen mammography is also influenced by the experience of the radiologist, with experienced radiologists having the highest sensitivity in diagnosing breast cancer [14]. Finally, the ionizing nature of the $\mathrm{X}$-ray mammography limits its frequent use.

To overcome some of these problems associated with mammography, a number of new technologies are currently being explored. Investigators have utilized alternative breast imaging methods, such as magnetic resonance imaging (MRI) and conventional ultrasound for imaging breast microcalcifications. Scintimammography and contrast-enhanced MRI are not sufficiently sensitive to detect clinically significant microcalcifications [15]. Similarly, conventional medical ultrasound imaging is not currently considered sufficiently reliable to visualize breast microcalcifications [16]-[18]. Microcalcification visualization by ultrasound is limited by a number of factors specific to this technique, such as speckle noise [18]. Although ultrasound can be used as an effective adjunct to mammography in detecting breast cancer [19], this technique will miss occult cancers where microcalcification may be the only sign of malignancy [20]. Thus, a sensitivity of only $63 \%$ was found in a study by Kasumi [21]. However, Yang, et al. 

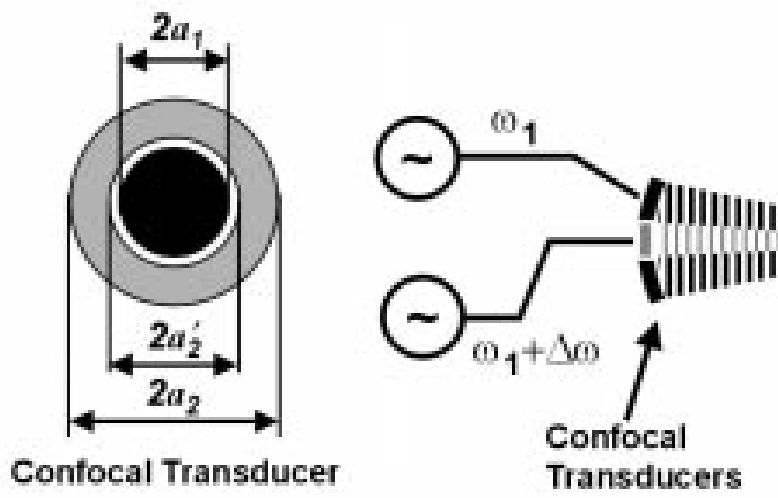

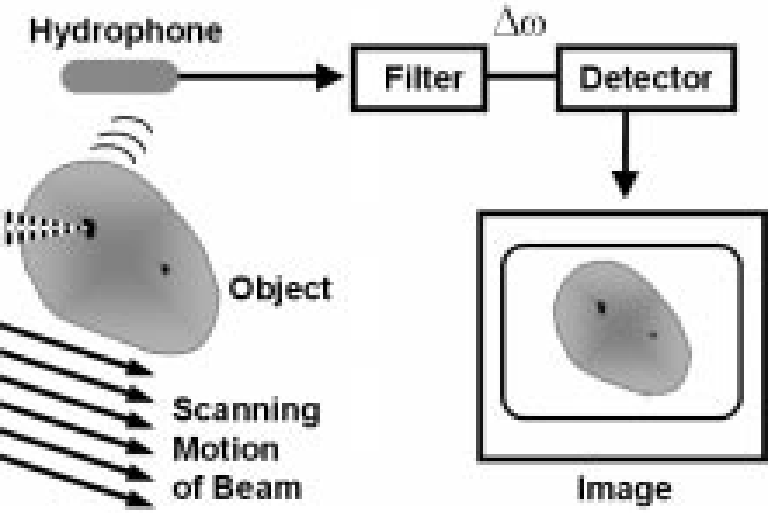

Fig. 1. Vibro-acoustography system diagram. The system includes a focused confocal transducer, (details shown on the left) consisting of a center disk and an outer ring. Two continuous wave generators drive these elements at slightly different frequencies. The transducer is focused on the object, with the beams interacting at the joint focal point to produce an oscillating radiation force on the object at the difference frequency. This force causes the object to vibrate and as a result an acoustic emission field is produced in the surrounding medium. This field is detected by the hydrophone and filtered by a bandpass filter centered at the difference frequency. The amplitude of the resulting signal, detected by the detector, is used to modulate the intensity of the image at a point corresponding to the position of the beam on the object. The image is formed by raster scanning of the object. The experiments take place in a water tank (not shown) containing the transducer, hydrophone and the object. The object, such as a tissue specimen, is secured on a latex sheet attached to a bracket for the scanning process.

[22] reported that using high-resolution ultrasound and X-ray mammography as the gold standard resulted in a sensitivity of $95 \%$ and specificity of $87 \%$ and accuracy of $91 \%$ in the detection of microcalcifications when these calcifications are within a mass lesion [22]. It should be noted that the presence of a mass lesion and its appearance in the ultrasound image could have been instrumental in the localization and identification of microcalcifications in such studies.

Microcalcifications are mainly composed of hydroxyapatite [18], [23], which is a very hard material compared with breast tissues. Therefore, an imaging modality that is sensitive to the elastic properties of tissues would likely be suitable for detection of microcalcifications. This group of imaging techniques is called elasticity imaging [24]-[37].

The general approach in elasticity imaging is to measure the response of tissue to an excitation force. An interesting strategy for producing the necessary excitation is to use the radiation force of ultrasound. This approach allows one to generate the force directly inside an organ. Several researchers have previously investigated the use of ultrasound radiation pressure for tissue characterization purposes. Sugimoto et al. [38] presented a method to measure tissue hardness by using the radiation force of a single ultrasound beam. In this method, the impulsive radiation force was used to generate localized deformation of tissue. Resulting transient deformation was measured as a function of time by the ultrasound Doppler method. Nightingale et al. [39] have studied the radiation force of ultrasound in a method named "remote palpation" to evaluate lesions in breast. They also used the ultrasound pulse-echo technique to detect tissue displacement. None of the above methods [38], [39] are specifically applied to the detection of breast microcalcifications. Walker [40] evaluated the deformation of soft tissues, including breast, by the radiation force of ultrasound. It should be noted that motion detection by ultrasound techniques (at diagnostic frequencies) becomes technically difficult if the motion is less than $1 \mu \mathrm{m}$. Walker predicted that the displacement produced by absorption of a continuous wave ultrasound beam of moderate intensity $\left(1 \mathrm{~W} / \mathrm{cm}^{2}\right)$ in breast tissue is too small (less than $0.01 \mu \mathrm{m}$ ) for consistent ultrasound detection.

Vibro-acoustography is a new imaging method based on the radiation force of ultrasound [41], [42]. This method is particularly useful in detecting hard inclusions in soft material. For example, vibro-acoustography has been used to image calcifications in human arteries [43]-[45]. A comparative study of vibro-acoustography with other radiation force methods for tissue elasticity imaging is presented in [46]. The spatial resolution of vibro-acoustography is in the submillimeter range, which makes the technique suitable for imaging microcalcifications [47], [48].

In this paper, we present an application of vibro-acoustography for detection of breast microcalcifications. We may call the new method vibro-acoustic tissue mammography. We compare images of human breast tissue specimens obtained by vibro-acoustography with high-resolution X-ray tissue mammography and validate the presence and position of calcifications in the tissue histologically.

\section{Methods AND MATERIALS}

\section{A. Theory of Radiation Force}

Radiation force is generated by a change in the energy density of an incident acoustic field. Consider a collimated ultrasound beam interacting with an object. The radiation force arising from this interaction has a component, $F$, in the beam direction. This component is proportional to the time average energy density of the incident wave $\langle E\rangle$ and the projected area of the object, $S$, as

$$
F=d_{r} S\langle E\rangle
$$

where $d_{r}$ is the drag coefficient and is a function of the scattered and absorbed power by the object. For the simple case of a reflecting plane target, $d_{r}$ is proportional to the power reflection coefficient. In vibro-acoustography [17], this force is used for imaging. This is accomplished by probing the object 
point-by-point. This technique ideally requires the stress field to be confined to a point, while its amplitude oscillates at selected frequencies.

To generate a localized oscillatory stress field, two intersecting continuous-wave focused ultrasound beams of different frequencies are used. It is only in the intersection region that the ultrasound field energy density is sinusoidally modulated and hence, the field can generate an oscillatory radiation force by interacting with the object.

The ultrasound beams can be shaped in a variety of ways for this purpose. An interesting configuration that results in a radially symmetric modulated field is obtained when two coaxial, confocal transducer elements are used (Fig. 1). In this case, we consider a two-element spherically focused annular array, consisting of a central disc with the radius of $a_{1}$ and an outer ring with the inner radius of $a_{2}^{\prime}$ and the outer radius of $a_{2}$. The common focal length of the elements is $z_{0}$. We also assume that the elements are excited by two $\mathrm{CW}$ signals at frequencies $\omega_{1}$ and $\omega_{2}=\omega_{1}+\Delta \omega$. Let us assume that the beams are propagating in the $z$ direction with the joint focal point at $z=0$. The resultant field on the $z=0$ plane may be written as

$$
p(t)=P_{1}(r) \cos \left[\omega_{1} t+\psi_{1}(r)\right]+P_{2}(r) \cos \left[\omega_{2} t+\psi_{2}(r)\right]
$$

where $P_{1}(r)$ and $P_{2}(r)$ are the pressure radial profiles in $r$ direction with $\psi_{1}(r)$ and $\psi_{2}(r)$ being the associated phase functions across the focal plane. For the transducer described above, these amplitude functions can be written as [31]

$$
\begin{aligned}
P_{1}(r)= & \rho c U_{01} \frac{\pi a_{1}^{2}}{\lambda_{1} z_{0}} \mathrm{jinc}\left(\frac{r a_{1}}{\lambda_{1} z_{0}}\right) \\
& \text { and } \\
P_{2}(r)= & \rho c U_{02} \frac{\pi}{\lambda_{2} z_{0}} \\
& \times\left[a_{2}^{2} \mathrm{jinc}\left(\frac{r a_{1}}{\lambda_{1} z_{0}}\right)-a_{2}^{\prime 2} \mathrm{jinc}\left(\frac{r a_{2}^{\prime}}{\lambda_{2} z_{0}}\right)\right]
\end{aligned}
$$

where $\lambda_{i}=2 \pi / \omega_{i}$ for $i=1,2$, are ultrasound wavelengths at the $i$ th transducer element $U_{0 i}, i=1,2$, is the velocity amplitude at the corresponding element. Also, jinc $(X)=J_{1}(X) / \pi X$, where $J_{1}(X)$ is the first-order Bessel function of the first kind. For well-focused beams, $P_{1}(r)$ and $P_{2}(r)$ diminish quickly away from the origin.

It can be shown that the short time average of the acoustic energy density in the intersection region has slow variations at frequency $\Delta \omega$ about its long time average (mean). Denoting this low-frequency component by $e_{\Delta \omega}(t)$, we can write

$$
e_{\Delta \omega}(t)=\frac{P_{1}\left(r_{0}\right) P_{2}\left(r_{0}\right)}{\rho c^{2}} \cos \left[\Delta \omega t+\Delta \psi\left(r_{0}\right)\right]
$$

where $\rho$ is the density, $c$ is the sound speed and $\Delta \psi=\psi_{2}(r)-$ $\psi_{1}(r)$. Now, consider a planar target on the focal plane. Referring to (1) and considering that the average energy density is position and time dependent in this case, the normal component of the time-varying force on an area element $d S$ at $r=r_{0}$ with a local drag coefficient $d_{r}\left(r_{0}\right)$ is

$$
f_{\Delta \omega}\left(r_{0}, t\right)=\frac{P_{1}\left(r_{0}\right) P_{2}\left(r_{0}\right)}{\rho c^{2}} \cos \left[\Delta \omega t+\Delta \psi\left(r_{0}\right)\right] d_{r}\left(r_{0}\right) d S .
$$

This function also represents the distribution of force, or the stress field, on the focal plane. The total force on the object can be found by integrating the above incremental force over the projection area $S$ on the focal plane

$$
\bar{f}_{\Delta \omega}\left(r_{0}, t\right)=\left|\bar{F}_{\Delta \omega}\right| \cos (\Delta \omega t+\Delta \bar{\psi})
$$

where $\bar{F}_{\Delta \omega}$ is the complex amplitude of the total force and $\Delta \bar{\psi}$ is the associated phase. For a well-focused beam $S$ is very small and hence, the force can be thought of as an oscillating point-like force applied to the object at the origin.

\section{B. Vibro-Acoustography System}

To explain the imaging method, we consider an oscillating point force, $\bar{F}_{\Delta \omega}$, applied to a point in the object. This force vibrates the object. Object vibrations result in the emission of an acoustic field in the surround energy that can be detected by a microphone (or hydrophone in water). The system diagram is shown in Fig. 1. The complex amplitude of acoustic emission pressure field, $P_{\Delta \omega}$, can be written as [17]

$$
P_{\Delta \omega}=\rho c^{2} H_{\Delta \omega}(l) Q_{\Delta \omega} \bar{F}_{\Delta \omega}
$$

where $Q_{\Delta \omega}$ is a complex function representing the mechanical frequency response of the object at this point $H_{\Delta \omega}(l)$ represents the combined frequency response of the propagation medium and the microphone located at distance $l$ from the object. It is assumed that $H_{\Delta \omega}(l)$ is unchanged for any target point in the object. Now, by scanning the object at a fixed-frequency $\Delta \omega$ and recording the acoustic emission signal, one can obtain the spatial distribution of $Q_{\Delta \omega} F_{\Delta \omega}$ (within a constant multiplier) which can be mapped into an image, displaying object morphology.

The point-spread-function (PSF) of this system is defined as the image of a point target located on the focal plane [17]. This function is proportional to the radiation stress field on the focal plane. This function can be found in an analytical form by combining (3), (4), and (6). The amplitude of this function can be written as [17]

$$
\begin{aligned}
h(r) & =\frac{1}{a_{2}^{2}-a_{2}^{\prime 2}} \operatorname{jinc}\left(\frac{r a_{1}}{\lambda_{1} z_{0}}\right) \\
& \times\left[a_{2}^{2} \operatorname{jinc}\left(\frac{r a_{2}}{\lambda_{2} z_{0}}\right)-a_{2}^{\prime 2} \operatorname{jinc}\left(\frac{r a_{2}^{\prime}}{\lambda_{2} z_{0}}\right)\right] \exp (j \Delta \bar{\psi}) .
\end{aligned}
$$

Assuming a 3-MHz-transducer with the outer diameter of 45 $\mathrm{mm}$, inner disk diameter of $29.6 \mathrm{~mm}$, and a gap of $2 \mathrm{~mm}$ between the inner disk and the outer ring and a focal length of $70 \mathrm{~mm}$, the amplitude of the resulting PSF function for $\Delta f=\Delta \omega / 2 \pi=$ $7.3 \mathrm{kHz}$ is shown in Fig. 2. The spatial resolution of the system, defined by the diameter of the central lobe, is about $0.7 \mathrm{~mm}$. The depth resolution (or the focal depth) of this transducer was experimentally determined. For this purpose, a small glass bead was scanned at different depths. The depth resolution, defined as the $-6-d B$ width of the response in the axial direction, was 


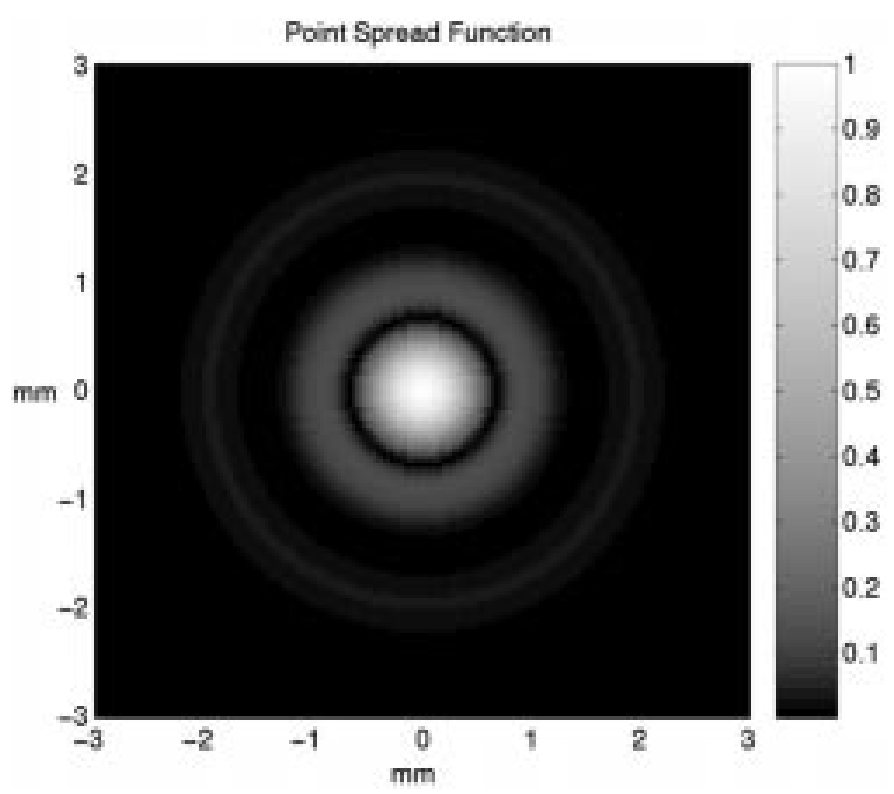

Fig. 2. Theoretical point spread function of the vibro-acoustography system.

found to be about $9 \mathrm{~mm}$. The peak response occurred when the bead was located at the focal distance from the transducer.

\section{Experimental Setup}

The experimental setup is shown in Fig. 1. The experiments were conducted in a water tank. (In a system designed for in vivo imaging the transducers can be placed in contact with the skin instead of using water. The hydrophone would also be placed in contact with the skin. Because at low frequencies the sound wave propagates almost uniformly in all directions, hydrophone position is not critical, as long as it is relatively close to the exposure site but not in the ultrasound path.) A two-element confocal ultrasound transducer array was positioned such that the beams meet the object at their joint focal point. Transducer specifications are as those in the previous section. The elements were driven by two stable radio-frequency (RF) synthesizers (HP $33120 \mathrm{~A}$ ) at frequencies of $3 \mathrm{MHz}$ and $3 \mathrm{MHz}+\Delta f$ Sound produced by the object vibration was detected by a submerged hydrophone (ITC model 680) placed within the water tank. The received signal was filtered and amplified by a programmable filter (Stanford Research Systems, SR650) to reject the noise, then digitized by a 12-bits/sample digitizer (National Instruments VXI-1000) at a rate sufficiently higher than the Nyquist rate. Data are recorded on a computer disc.

\section{Experiment Procedure}

To evaluate the capability of the system for imaging small particles about the size of a common breast microcalcification, we constructed a test object comprised of four small glass beads, ranging from $260 \mu \mathrm{m}$ to $400 \mu \mathrm{m}$ in diameter imbedded in a block of tissue- mimicking gel. The resulting vibro-acoustography image is shown in Fig. 3, demonstrating the capability of the system in detecting and imaging beads that are at least $260 \mu \mathrm{m}$ in diameter.

Tissue experiments were conducted on excised human breast tissue samples. These tissues were obtained postsurgically and

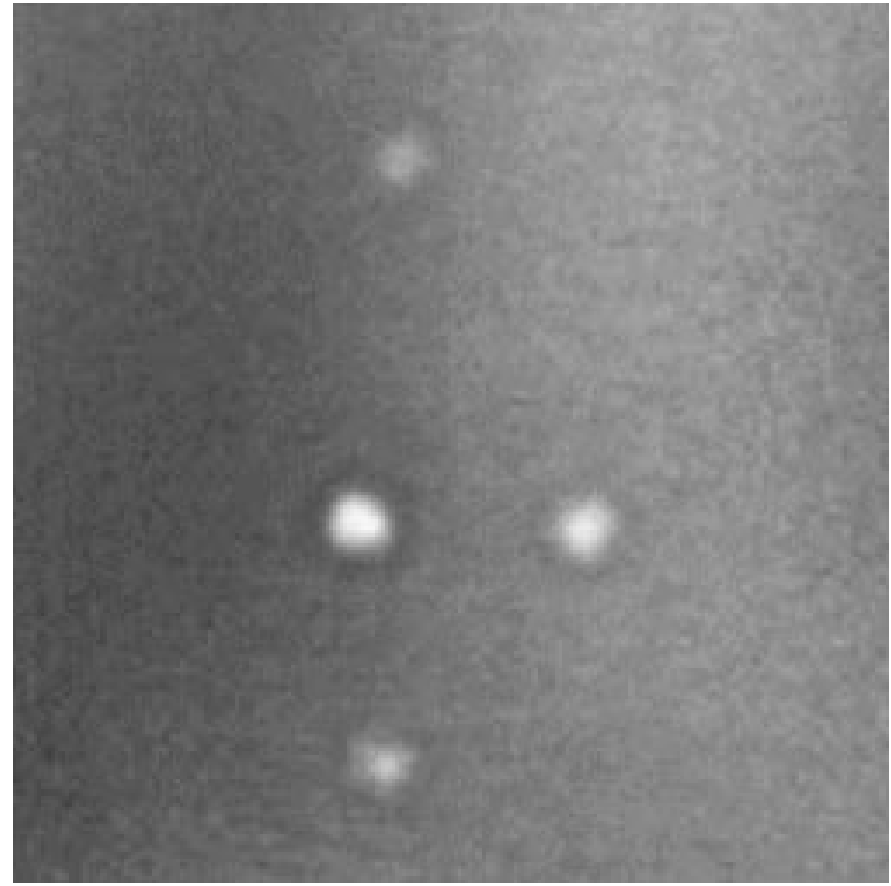

Fig. 3. Image of glass beads in gel. This image is obtained by scanning the gel phantom. This image covers an area of $20 \mathrm{~mm}$ by $20 \mathrm{~mm}$, scanned at 0.2 $\mathrm{mm} /$ pixel. The beads are located about $10 \mathrm{~mm}$ deep inside the gel. The diameter of the top bead is about $260 \mu \mathrm{m}$ and the diameter of the largest bead is about $400 \mu \mathrm{m}$. The vibration frequency was set at $53.5 \mathrm{kHz}$.

were fixed in formaldehyde for at least three days and not more than six months at the time of experiments. It is known that formaldehyde tends to harden soft tissue and, thus, may introduce an additional variable in the experiments. However, because our main target (microcalcifications) is much harder than the fixed tissue, we did not consider tissue-hardening phenomenon as a major problem in our experiments. Patients' records were reviewed to identify tissues with high probability of having microcalcifications. Selected tissues were cut into (approximately) $3 \times 3 \mathrm{~cm}$ and 3 - to 5 -mm-thick pieces and imaged using a high-resolution $\mathrm{X}$-ray mammography machine. Therefore microcalcifications were at most $5 \mathrm{~mm}$ from the front surface of the sample. The X-ray mammograms were then read to identify presence of microcalcifications. Tissue pieces identified with microcalcification were each mounted flat with a few droplets of glue on a latex sheet and secured in a scanning bracket. This bracket is designed to hold the tissue piece in the water for acoustic scanning. Glue drops were carefully placed on areas away from the region with microcalcifications. Small pieces of suture were sewed to the tissue and used as identification marks. For this purpose, small knots are placed at different positions on the tissue away from microcalcifications. These markers could be seen in vibro-acoustography, X-ray (often very dim) and photographic images.

Specimen X-ray mammographic images were obtained from each mounted sample. Each X-ray image was used as a reference for comparison with the corresponding vibro-acoustography image. The system illustrated in Fig. 1 was used to obtain vibro-acoustography images of tissue samples. The scanning process was performed by a raster motion of the specimen, the ultrasound beam being perpendicular to the large flat surface 


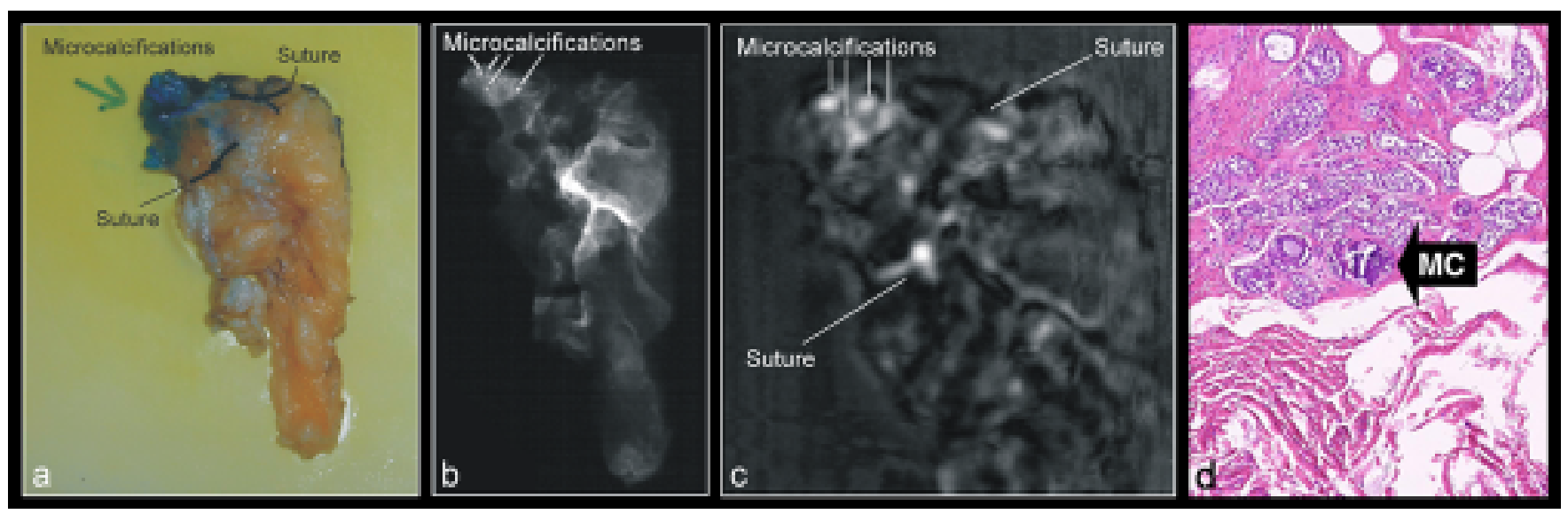

Fig. 4. Vibro-acoustography of the first specimen. (a) Photograph of a breast specimen mounted on the scanning bracket, (b) The X-ray mammogram of the selected tissue pieces mounted on the scanning bracket. Microcalcifications can be seen on the top left of this X-ray, (c) Vibro-acoustography image of the tissue at $25 \mathrm{kHz}$. Microcalcifications can be seen in this image as bright spots on the top left and (d) Histology of the tissue specimen around the region with some microcalcifications.

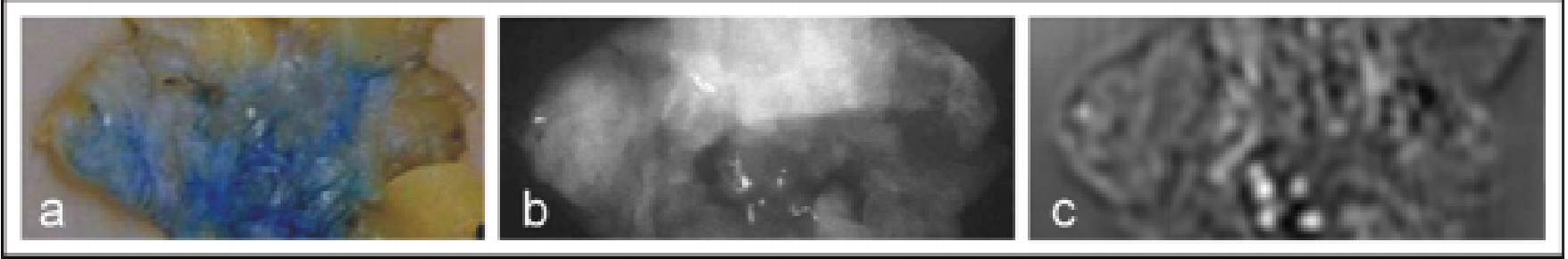

Fig. 5. Vibro-acoustography of the second specimen. (a) Photograph of the tissue specimen mounted on the scanning bracket. (b) X-ray mammogram of (a) showing some microcalcifications at the center of the image. (c) Vibro-acoustography of (a). Microcalcifications can be seen as bright spots at same location as in the X-ray.

of the tissue layer and passing through the 3- to 5-mm-thickness of the tissue. The specimen was placed on the focal plane, such that its entire thickness was within the focal depth of the transducer. Experiments were conducted in a water tank at room temperature (about $21^{\circ} \mathrm{C}$ ). Sections of the tissue identified with microcalcification were then cut for histologic study to identify microcalcifications and validate the imaging results.

\section{RESULTS}

Fig. 4(a) shows the photograph of a breast specimen mounted on the scanning bracket. Sutures are knotted on the tissue for identification purpose. Fig. 4(b) shows the X-ray mammogram of the selected tissue pieces mounted on the scanning bracket. The sutures are barely visible. Microcalcifications can be seen on the top left of this X-ray in a high-density sclerotic region. This sample was then scanned in the water tank. The vibroacoustography image of the tissue, at $\Delta f=25 \mathrm{kHz}$, is shown in Fig. 4(c). Microcalcifications can be seen in this image as bright spots. Note that the number of microcalcifications matches with the corresponding spots in the X-ray mammography. Also noticeable is that dense sclerotic tissue appears dim and does not interfere with identification of calcification in the vibro-acoustic image. Microcalcifications identified by vibro-acoustography were verified histologically. Fig. 4(d) shows the histology of the region around the calcifications. The breast parenchyma shows atypical lobular hyperplasia and microcalcifications in the lobules. We measured the size of the microcalcification at the lower left, by measuring the spot size in the X-ray mammogram and found to be approximately $110 \mu \mathrm{m}$ in diameter.

Fig. 5(a) shows the photograph of the second breast tissue specimen mounted on the scanning bracket. Fig. 5(b) is the $\mathrm{X}$-ray mammogram of this tissue and shows the microcalcifications at the center of the image. Fig. 5(c) shows the vibro-acoustic image of this tissue at $22 \mathrm{kHz}$. Microcalcifications can be seen as bright spots at the same location as in the $\mathrm{X}$-ray. The breast parenchyma histologically showed comedo type ductal carcinoma in situ, with calcifications in regions of comedo necrosis.

Fig. 6(a) shows the X-ray mammogram of another breast tissue specimen with a large microcalcification. Fig. 6(b) shows the corresponding vibro-acoustic image, with the microcalcification shown as a bright spot within the relatively dark background of soft tissue.

\section{DISCUSSION}

Experimental vibro-acoustography images presented in this report demonstrate two important facts: 1) The vibro-acoustography imaging method is capable of detecting small microcalcifications in breast tissue; 2) Microcalcifications can be delineated from within dense and sclerotic tissues. Results shown here also indicate that such images have high spatial resolution (700 $\mu \mathrm{m}$ ), the capability of detecting small (about $110 \mu \mathrm{m}$ in diameter and above) microcalcifications, no speckles, good contrast and high signal to noise ratio. 


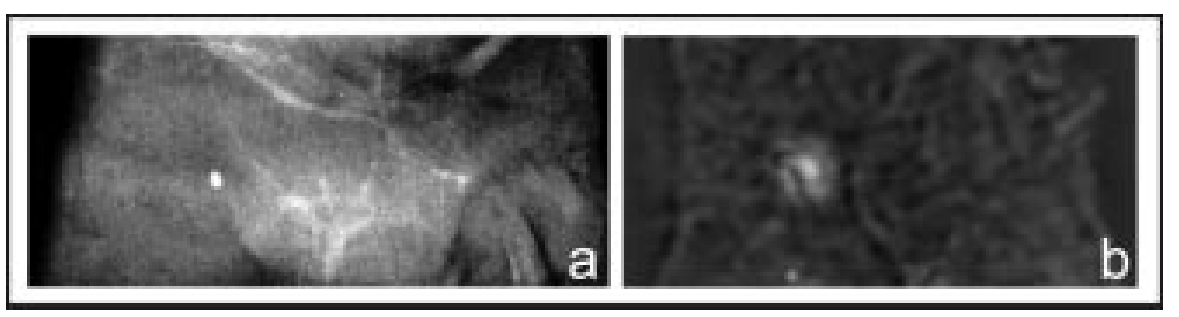

Fig. 6. Vibro-acoustography of the third specimen. (a) X-ray mammogram of the tissue sample showing a large microcalcification and (b) Vibro-acoustography image of (a).

A comparison between the vibro-acoustography image [Fig. 4(b)] and the corresponding X-ray mammography [Fig. 4(c)], reveals an interesting capability of vibro-acoustography method. High-density sclerotic tissue in the region surrounding the microcalcification absorbs a great deal of $\mathrm{X}$-ray energy; hence, the X-ray image shows very low contrast within this region and as a result microcalcifications are barely visible. However, the vibro-acoustography image delineates these calcifications with high contrast. This demonstrates that some tissues that are radiologically opaque may be transparent in vibro-acoustography images giving the potential to detect calcification in radiologically dense breasts.

The halo seen around the images of each microcalcification is an artifact of the vibro-acoustography system. This effect is seen more clearly in the PSF image shown in Fig. 2. Multiple rings around the center spot are due to the sidelobes of the Bessel functions that appear in the PSF function (9) as discussed in the previous section.

Another artifact is due to the glue used to bond the tissue specimens to the latex sheet. We used small droplets of instant glue (Krazy Glue brand, Elmers Products, Inc. Columbus, $\mathrm{OH}$ ) for this purpose. This type of glue produces a relatively strong acoustic emission making it visible in vibro-acoustography images. Fig. 7 shows a vibro-acoustography image of three droplets of this glue on a latex sheet, resembling images of breast microcalcification. To avoid mistaking glue droplets for microcalcifications, we marked the glued region of tissue and took extra precautions to locate these regions on the vibro-acoustography images.

The choice of vibration frequency $\Delta f$ used in each experiment was, to some extent, determined by the resonance frequencies of the water tank. The acoustic emission field resulting from vibrations of microcalcifications is very small. To facilitate the detection process, we often chose the vibration frequency to correspond to one of the resonance frequencies of the water tank. This way, the water tank acts as an amplifier to improve the signal-to-noise ratio (SNR). Because the human body does not have a regular geometric shape with parallel walls, such resonance phenomenon is not likely to take place. Therefore, the choice of $\Delta f$ may no longer be based on the resonance effect.

Ultrasound standing waves between the transducer and the object may cause artifacts in the image. This may happen if the object reflects a significant portion of the incident beam back to the transducer. The transducer surface can then act as a mirror to re-reflect the reflected field toward the object. This process may occur more than once. The resulting field at the object is the sum of the incident and all the reflected fields. De-

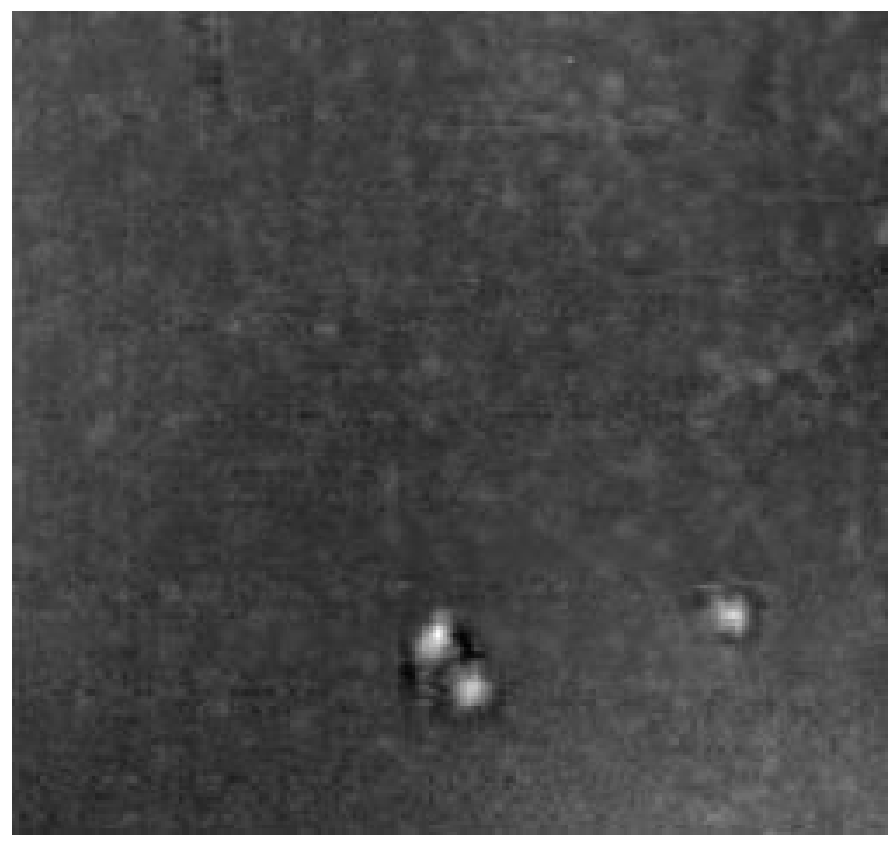

Fig. 7. Vibro-acoustography of the instant glue drops. The glue drops (about $1 \mathrm{~mm}$ in diameter) are placed on a latex sheet.

pending on the distance between the transducer and the object and the ultrasound- wavelength, those components may interact constructively or destructively. Hence, the field intensity and the resulting image can, therefore, become excessively sensitive to the transducer-to-object distance. The effect of standing wave phenomena on the image diminishes as the ultrasound bath loss increases. Therefore, it is expected that standing wave artifacts would be less of a problem in clinical applications of vibro-acoustography for in vivo breast imaging.

In the present study, we imaged tissue samples only in two dimensions (i.e., a single slice). However, the ultrasound beam used here produces a stress field that is confined in three dimensions. Therefore, it is possible, in principle, to selectively scan several slices of the object at different depths to produce a three-dimensional (volume) image of the object.

Application of the vibro-acoustography for breast imaging is both promising and challenging. An expected difficulty in using vibro-acoustography on patients is achieving a high SNR. Several factors that can reduce the SNR below what we have in the experimental system. These factors include limitation on the ultrasound intensity, body attenuation of the ultrasound beam, defocusing of the ultrasound beam due to phase aberrations, and the biological noise generated by the body. Imaging speed is 
another concern in in vivo vibro-acoustography. The scanning mechanism used in the experiment presented in this paper scan the object one point at a time, making the data collection a relatively lengthy process (up to a few minutes per image). A long imaging time is not desirable for breast imaging because body motions within this period can introduce "motion artifact " in the images.

There are several benefits in using vibro-acoustography for in vivo breast imaging as an alternative, or in parallel, to $\mathrm{X}$-ray mammography. One advantage of vibro-acoustography stems from the fact that it uses ultrasound energy, which can easily penetrate in dense tissues. Such tissues are normally radio-opaque and difficult to image with X-ray. Additionally, the present method is relatively safe and hence, may be useful in applications where the use of X-ray is limited to its ionizing effects. Vibro-acoustic detection and follow-up of microcalcifications has several clinical potentials, including the following.

- Detection of microcalcifications in the breast of women who are younger and/or have radiologically very dense breast. We showed that ultrasound can penetrate radiologically dense tissue and detect calcification.

- Detection of microcalcification in pregnant or lactating women.

- Detection of microcalcifications in women with radio-opaque breast implants.

\section{SUMMARY}

In this paper, we present a noninvasive imaging method for detecting microcalcifications in breast tissue. This method uses ultrasound in a fundamentally new way to image the tissue at low ( $\mathrm{kHz}$ range) frequencies, producing high signal-to-noise and speckle-free images.

The spatial resolution of vibro-acoustic images is determined by the focal beamwidth of the two-element transducer, which was in the order of $0.7 \mathrm{~mm}$ for the transducer used here. Experiments were conducted on human breast tissue specimens. $\mathrm{X}$-ray tissue mammography images were used for initial identification of microcalcifications and also as reference images for comparison with the corresponding vibro-acoustography images. Tissue histology was also used to validate presence of microcalcification.

Results indicate that microcalcifications associated with a variety of breast pathology, including those located in high-density or sclerotic regions of tissue, can be detected by vibro-acoustography. Microcalcifications as small as $110 \mu \mathrm{m}$ in diameter were detected by vibro-acoustic tissue mammography.

Further development of vibro-acoustic tissue mammography may lead to a novel imaging tool for applications. This method can be considered as a nonionizing alternative to conventional $\mathrm{X}$-ray mammography and may be useful for such cases as pregnant patients, where the use of ionizing radiation is not allowed. Also, vibro-acoustography is potentially useful for imaging dense breast where the use of conventional X-ray mammography may be limited.

\section{ACKNOWLEDGMENT}

The authors are grateful to the following individuals for their valuable work during the course of this study: Dr. R. E. Johnson for valuable comments on the clinical aspects of microcalcification in breast, T. Distad for providing postsurgical tissue specimens, M. Siewert for providing tissue X-rays, Dr. M. Morton for reading X-ray mammograms, R. Kinnick for laboratory support and scanning tissues, D. Cook for scanning some of the tissues, Dr. K. Kanehira for histologic evaluation of tissues, J. Patterson for graphic support and E. Quarve for secretarial assistance.

\section{REFERENCES}

[1] M. D. Abeloff, A. S. Lichter, J. E. Niederhuber, L. J. Pierce, and R. R. Love, "Breast," in Clinical Oncology, 2nd ed, M. D. Pierce, Ed. New York: Churchill Livingstone, 2000, ch. 75, pp. 2051-2138.

[2] S. C. Lester and R. S. Cortan, "The breast," in Robins Pathologic Basis of Diseases, 6th ed, V. Kumar, T. Collins, and T. Collins, Eds. Philadelphia, PA: Saunders, 1999, pp. 1093-1117.

[3] G. D. Dodd, "American cancer society guidelines on screening for breast cancer," Cancer, vol. 69, no. 7, pp. 1885-1887, 1992.

[4] J. V. Fioricia, "The breast," in Danforth Obstetrics and Gynecology, 8th ed, J. R. Scott, P. J. Di Saia, C. B. Hammond, and W. M. Spellacy, Eds. Philadelphia, PA: Lippincott Williams and Williams, 1999, ch. 40.

[5] "Screening recommendations of the forum panel," J. Gerontol., no. 5, p. 47, Nov. 1992.

[6] S. W. Fletcher, W. Black, and R. Harris et al., "Report of the international workshop on screening for breast cancer," J. Nat. Cancer Inst., vol. 85, no. 30, pp. 1644-1656, 1993.

[7] K. Kerlikowske, "Effect of age, breast density and family history on the sensitivity of first screen mammography," JAMA, vol. 276, no. 1, pp. 33-37, July 1996

[8] C. D. Lehman, E. White, S. Peacock, and M. J. Drucker, "Urban N. effect of age and breast density on screening mammograms with false-positive findings," Amer. J. Roentgenol., vol. 173, no. 6, pp. 1651-1655, Dec. 1999.

[9] C. J. Baines, M. Vidmar, G. McKeown-Eyssen, and R. Tibshirani, "Impact of menstrual phase on false-negative mammograms in the canadian national breast screening study," Cancer, vol. 80, no. 4, pp. 720-724, August 1997.

[10] E. White, P. Velentgas, and M. T. Mandelson et al., "Variation in mammographic breast density by time in menstrual cycle among women aged 40 to 49 years," J. Nat. Cancer Inst., vol. 90, no. 12, pp. 906-910, June 1998.

[11] M. B. Laya, D. B. Larson, S. H. Taplin, and E. White, "Effect of estrogen replacement therapy on the specificity and sensitivity of screening mammography," J. Nat. Cancer Inst., vol. 88, no. 10, pp. 643-649, 1996.

[12] J. C. Litherland, S. Scotland, D. Hole, and C. Cordiner, "The effect of hormone replacement therapy on the sensitivity of screening mammograms," Clin. Radiol., vol. 54, no. 5, pp. 285-288, May 1999.

[13] A. M. Kavanagh, H. Mitchel, and G. G. Giles, "Hormone replacement therapy and accuracy of mammographic screening," Lancet, vol. 355, no. 9200 , pp. 270-274, Jan. 2000.

[14] L. Liberman, A. F. Abramson, and F. B. Squires et al., "The breast imaging reporting and data system-positive predictive value of mammographic features and final assessment categories," Amer. J. Roentgenol., vol. 171, no. 1, pp. 35-40, July 1998.

[15] R. R. Tiling, I. Khalkhali, H. Sommer, R. Link, R. Moser, F. Willemen, T. Pfluger, K. Tats, and K. Hahn, "Limited value of scintimammography and contrast-enhanced MRI in the evaluation of microcalcification detected by mammography," Nucl. Med. Common, vol. 19, no. 1, pp. 55-62, Jan. 1998.

[16] A. J. Potterton, D. J. Peakman, and J. R. Young, "Ultrasound demonstration of small breast cancers detected by mammographic screening," Clin. Radiol., vol. 49, pp. 808-813, Nov. 1994.

[17] P. B. Guyer, K. C. Dewbury, D. Warwick D, J. Smallwood, and I. Taylor, "Direct contact B-scan clinical ultrasound in the diagnosis of solid breast masses," Clin. Radiol., vol. 37, no. 5, pp. 451-458, Sept. 1986.

[18] M. E. Anderson, M. S. Soo, R. C. Bentley, and G. E. Trahey, "The detection of breast microcalcifications with medical ultrasound," J. Acoust. Soc. Amer, vol. 101, no. 1, pp. 29-39, Jan. 1997. 
[19] H. M. Zonderland, E. G. Cokerkamp, J. Hemans, M. J. van de Vijver, and A. E. Van Voorthuisen, "Diagnosis of breast cancer: Contribution of US as an adjunct to mammography," Radiol., vol. 213, no. 2, pp. 413-422, Nov. 1999.

[20] E. A. Sickles, "Sonographically detectability of breast calcifications," Proc. SPIE, vol. 419, pp. 51-52, 1983.

[21] F. Kasumi, "Can microcalcifications located within breast carcinomas be detected by ultrasound imaging?," Ultrasound Med. Biol., vol. 14, no. 1, pp. 175-82, 1988

[22] W. T. Yang, M. Suen, A. Ahuja, and C. Meterweli, "In vivo demonstration of microcalcification in breast cancer using high resolution ultrasound," Br. J. Radiol, vol. 70, pp. 685-690, July 1997.

[23] D. E. Grenoble, J. L. Katz, K. L. Dunn, K. L. Murty, and R. S. Gilmore, "The elastic properties of hard tissues and apatites," J. Biomed. Mater. Res., vol. 6, no. 3, pp. 221-233, May 1972.

[24] M. O'Donnell, A. R. Skovoroda, B. M. Shapo, and S. Y. Emelianov, "Internal displacement and strain imaging using ultrasonic speckle tracking," IEEE Trans. Ultrason. Ferroelect. Freq. Contr., vol. 1, pp. 314-325, May 1994.

[25] J. Ophir, I. Cespedes, H. Ponnekanti, Y. Yazdi, and X. Li, "Elastography: A quantitative method for imaging the elasticity of biological tissues," Ultrason. Imag., vol. 13, no. 2, pp. 111-134, April 1991.

[26] Y. Yamakoshi, J. Sato, and T. Sato, "Ultrasonic imaging of internal vibration of soft tissue under forced vibration," IEEE Trans. Ultrason. Ferroelect. Freq. Contr., vol. 37, pp. 45-53, Mar. 1990.

[27] T. A. Krouskop, D. R. Dougherty, and F. S. Vinson, "A pulsed doppler ultrasonic system for making noninvasive measurements of the mechanical properties of soft tissue," J. Rehab. Res. Dev., vol. 24, no. 2, pp. 1-8, 1987.

[28] R. M. Lerner, S. R. Huang, and K. J. Parker, "Sonoelasticity images derived from ultrasound signals in mechanically vibrated tissues," Ultrasound Med. Biol., vol. 16, no. 3, pp. 231-239, 1990.

[29] S. K. Alam, D. W. Richards, and K. J. Parker, "Detection of intraocular pressure change in the eye using sonoelastic doppler ultrasound," Ultrasound Med. Biol., vol. 20, no. 8, pp. 751-758, 1994.

[30] L. Gao, K. J. Parker, R. M. Lerner, and S. F. Levinson, "Imaging of the elastic properties of tissue-A review," Ultrasound Med. Biol., vol. 22, no. 8, pp. 959-977, 1996

[31] R. Muthupillai, D. J. Lomas, P. J. Rossman, J. F. Greenleaf, A. Manduca, and R. L. Ehman, "Magnetic resonance elastography by direct visualization of propagating acoustic strain waves," Science, vol. 269, no. 5232, pp. 1854-1857, Sept. 1995

[32] J. Ophir, S. K. Alam, B. Garra, F. Kallel, E. Konofagou, T. Krouskop, and T. Varghese, "Elastography: Ultrasonic estimation and imaging of the elastic properties of tissues," Proc. Inst. Mech. Eng. [H]-J Eng. Med., vol. 213, no. 3, pp. 203-233, 1999.

[33] D. Fu, S. F. Levinson, S. M. Gracewski, and K. J. Parker, "Non-invasive quantitative reconstruction of tissue elasticity using an iterative forward approach,” Phys. Med. Biol., vol. 45, no. 6, pp. 1495-509, June 2000.
[34] L. S. Taylor, B. C. Porter, D. J. Rubens, and K. J. Parker, "Three-dimensional sonoelastography: Principles and practices," Phys. Med. Biol., vol. 45, no. 6, pp. 1477-1494, June 2000.

[35] K. J. Parker, D. Fu, S. M. Graceswki, F. Yeung, and S. F. Levinson, "Vibration sonoelastography and the detectability of lesions," Ultrasound Med. Biol., vol. 24, no. 9, pp. 1437-1447, November 1998.

[36] D. D. Steele, T. L. Chenevert, A. R. Skovoroda, and S. Y. Emelianov, "Three-dimensional static displacement, stimulated echo NMR elasticity imaging," Phys. Med. Biol., vol. 45, no. 6, pp. 1633-1648, June 2000

[37] T. L. Chenevert, A. R. Skovoroda, M. O'Donnell, and S. Y. Emelianov, "Elasticity reconstructive imaging by means of stimulated echo MRI," Magn. Reson. Med., vol. 39, no. 3, pp. 482-490, Mar. 1998.

[38] S. Sugimoto, S. Ueha, and K. Itoh, "Tissue hardness measurement using the radiation force of focused ultrasound," in IEEE Ultrason. Symp. Proc., 1990, pp. 1377-1380.

[39] K. R. Nightingale, R. W. Nightingale, M. T. Palmeri, and G. E. Trahey, "A finite element model of remote palpation of breast lesions using radiation force: Factors affecting tissue displacement," Ultrason. Imag., vol. 22 , no. 1 , pp. 35-54, Jan. 2000.

[40] W.F. Walker, "Internal deformation of a uniform elastic solid by acoustic radiation force," J. Acoust. Soc. Amer, vol. 105, no. 4, pp. 2508-2558, Apr. 1999.

[41] M. Fatemi and J. F. Greenleaf, "Ultrasound stimulated vibro-acoustic spectroscopy," Science, vol. 280, pp. 82-85, Apr. 1998.

[42] — , "Vibro-acoustography: An imaging modality based on ultrasound stimulated acoustic emission," in Proc. Nat. Acad. Sci. USA, vol. 96, June 1999, pp. 6603-6608.

[43] J. F. Greenleaf, R. L. Ehman, M. Fatemi, and R. Muthupillai, "Imaging elastic properties of tissue," in Ultrasound in Medicine, F. A. Duck, A. C. Baker, and H. C. Starritt, Eds. Philadelphia, PA: Inst. Phys., 1998, ch. 14.

[44] M. Fatemi and J. F. Greenleaf, "Probing the dynamics of tissue at low frequencies with the radiation force of ultrasound," Phys. Med. Biol., vol. 45, pp. 1449-1464, 2000.

[45] _ - "Imaging the viscoelastic properties of tissue," in Topics in Applied Physics, M. Fink, J.-P. Montagner, and A. Tourin, Eds. Berlin, Germany: Springer-Verlag, 2001, vol. 82, to be published.

[46] _ - "Imaging and evaluating the elastic properties of biological tissues," BMUS Bulletin, vol. 8, no. 4, pp. 16-18, Nov. 2000

[47] — , "Vibro-acoustography system modeling," in Era of Hope Proc., vol. I, June 2000, p. 192

[48] P. M. Morse and K. U. Ingard, Theoretical Acoustics. New York: McGraw-Hill, 1968, p. 192. 